\title{
Thermoelectric and thermo-Coulomb effects in tunnel junctions
}

\author{
M. Amman \\ Department of Physics, University of Michigan, Ann Arbor, MI 48109, USA \\ E. Ben-Jacob \\ School of Physics and Astronomy, Raymond and Beverly Sackler Faculty of Exact Sciences, Tel-Aviv University, \\ 69978 Tel-Aviv, Israel
}

and

J.L. Cohn

Naval Research Laboratories, Washington, DC 20375, USA

Received 9 July 1991; revised manuscript received 5 October 1992; accepted for publication 12 October 1992 Communicated by A.A. Maradudin

\begin{abstract}
We present a study of the thermoelectric effects in tunnel junctions. In particular we calculate the thermoelectric power coefficient $S$ and the Peltier coefficient $\Pi$. For macroscopic junctions we demonstrate the sensitivity of $S$ and $\Pi$ to the structure of the density of states. For mesoscopic junctions we show that Coulomb effects modify the ordinary Onsager picture and the relation $\Pi=-T S$. The coefficients $S$ and $\Pi$ are found to be very sensitive to the coupling of the junctions to the external world. We comment on the relevance of these effects to scanning tunneling microscope measurements.
\end{abstract}

\section{Introduction}

During the last five years considerable interest has been directed towards tunnel junction systems where the discreteness of the electronic charge plays a prominent and observable role [1]. These systems involve at least one ultra-small conductive element with a capacitance $C$ such that its charging energy $E_{\mathrm{C}}$ is larger than the thermal energy,

$E_{\mathrm{C}} \equiv \frac{e^{2}}{2 C} \gg k_{\mathrm{B}} T$.

The theoretical and experimental study spans from the simplest element - a single mesoscopic normal junction - to systems of 1-D and 2-D arrays of junctions [2,3]. New phenomena, such as the Coulomb blockade, Coulomb staircase, and single charge solitons have been predicted and observed. All these phenomena depend on the electric response of the systems.

Being mesoscopic systems, their electric response is crucially sensitive to the nature of the external driving circuit [4,5]. Motivated by the new understanding, we expect that the thermal response of the junctions will show phenomena analogous to those studied in the electrical response, while new phenomena will be observed in the thermoelectric response [6].

As a first step in this direction, we present here a study of two thermoelectric effects, the Seebeck and Peltier effects [7], in mesoscopic normal junctions assuming that heat is transferred only by the tunneling charges. 
We show that the temperature dependence of the thermoelectric power coefficient $S$ and the Peltier coefficient $\Pi$ is very sensitive to the nature of the external circuit. Moreover, the ordinary Onsager picture [7] has to be modified as well as the relation $\Pi=-T S$.

\section{The thermoelectric power and the Peltier coefficients}

The Seebeck effect is the development of a voltage $V$ across an open junction (zero electric current) when a temperature difference of $\Delta T$ is applied. The operational definition of the thermoelectric power coefficient $S$ is

$S \equiv \lim _{\Delta T \rightarrow 0} \frac{V}{\Delta T}$,

where $V$ is the measured voltage across the open junction (infinite external resistance).

Alternatively, $V$ is defined as the applied voltage required to balance the current due to the temperature difference. For macroscopic junctions, both definitions yield the same result. Moreover, within linear response theory (applicable for macroscopic tunnel junctions), the electric current passed through the junction is

$\left.I(V, \Delta T) \approx \lim _{\Delta T \rightarrow 0} \frac{\partial I}{\partial \Delta T}\right|_{V=0} \Delta T+\left.\lim _{V \rightarrow 0} \frac{\partial I}{\partial V}\right|_{\Delta T=0} V$.

Hence, the thermoelectric power coefficient for $I(V, \Delta T)=0$ may be expressed as

$S=-\frac{\lim _{\Delta T \rightarrow 0} \partial I /\left.\partial \Delta T\right|_{V=0}}{\operatorname{tim}_{V \rightarrow 0} \partial T /\left.\partial\right|_{\Delta T=0}}$,

which is the form typically used for calculations.

The Peltier effect refers to the heat current $I_{\mathrm{Q}}$ that passes across the junction when the two sides are kept at equal temperature and an electric current $I$ is forced to flow. Accordingly, the Peltier coefficient $I$ is defined as

$\left.\Pi \equiv \lim _{I \rightarrow 0} \frac{\partial I_{\mathrm{Q}}}{\partial I}\right|_{\Delta T=0}$.

Within linear response theory, using the relation $\partial I / \partial V=(\partial V / \partial I)^{-1}, \Pi$ is given by

$\Pi=\frac{\lim _{V \rightarrow 0} \partial I_{\mathrm{Q}} /\left.\partial V\right|_{\Delta T=0}}{\operatorname{tim}_{V \rightarrow 0} \partial I /\left.\partial V\right|_{\Delta T=0}}$,

which has a form equivalent to that of eq. (4). Using the Onsager relations for the off-diagonal terms [7],

$\left.\lim _{V \rightarrow 0} \frac{\partial I_{\mathrm{Q}}}{\partial V}\right|_{\Delta T=0}=\left.T \lim _{\Delta T \rightarrow 0} \frac{\partial I}{\partial \Delta T}\right|_{V=0}$,

we obtain the following relation,

$\Pi=-T S$.

Note that, similarly to eq. (5), we can obtain

$S=\left.\lim _{I \rightarrow 0} \frac{\partial I_{\mathrm{S}}}{\partial I}\right|_{\Delta T=0}$, 
which is a motivation to view $S$ as the measure of the entropy transferred per carrier.

\section{The effect of the density of states}

We now consider macroscopic junctions, so that the charging energy contribution may be ignored and the linear response definitions of the thermoelectric power coefficient and the Peltier coefficients are applicable. For such a tunnel junction, the electric current passed through it is $I=e(r-l)$, where the electron tunneling rates from the right and left, respectively, are [8]

$$
\begin{aligned}
& r=\frac{1}{e^{2} R} \int_{-\infty}^{\infty} D_{l}(E) D_{r}(E-e V) f^{T}(E-e V)\left[1-f^{T+\Delta T}(E)\right] \mathrm{d} E, \\
& l=\frac{1}{e^{2} R} \int_{-\infty}^{\infty} D_{l}(E) D_{r}(E-e V) f^{T+\Delta T}(E)\left[1-f^{T}(E-e V)\right] \mathrm{d} E .
\end{aligned}
$$

Here $f^{T}(E)=1 /\left[\exp \left(E / k_{\mathrm{B}} T\right)+1\right]$ is the Fermi-Dirac distribution function, $D_{r}(E)$ and $D_{l}(E)$ are the right and left electrode normalized density of states (DOS) ${ }^{\# 1}$, and $R$ is the normal state resistance of the junction, which includes the normalization factors of $D_{r}(E)$ and $D_{l}(E)$.

The partial derivatives of the current with respect to the temperature difference and the voltage are given by

$$
\begin{aligned}
& \left.\lim _{\Delta T \rightarrow 0} \frac{\partial I}{\partial \Delta T}\right|_{V=0}=\frac{1}{e R} \int_{-\infty}^{\infty} D_{l}(E) D_{r}(E) \frac{E}{T} \frac{\partial f^{T}(E)}{\partial E} \mathrm{~d} E, \\
& \left.\lim _{V \rightarrow 0} \frac{\partial I}{\partial V}\right|_{\Delta T=0}=\frac{1}{R} \int_{-\infty}^{\infty} D_{l}(E) D_{r}(E)\left(-\frac{\partial f^{T}(E)}{\partial E}\right) \mathrm{d} E .
\end{aligned}
$$

For normal metal tunnel junctions we evaluate the integrals using the Sommerfeld expansion:

$$
\int_{-\infty}^{\infty} G(E)\left(-\frac{\partial f^{T}(E)}{\partial E}\right) \mathrm{d} E \approx G(0)+\frac{1}{6} \pi\left(k_{\mathrm{B}} T\right)^{2} G^{\prime \prime}(0)
$$

and obtain

$$
\begin{aligned}
& \left.\lim _{\Delta T \rightarrow 0} \frac{\partial I}{\partial \Delta T}\right|_{V=0} \approx-\frac{\pi^{2} k_{\mathrm{B}}^{2} T}{3 e R}\left[D_{l}(0) D_{r}(0)\right]^{\prime}, \\
& \left.\lim _{V \rightarrow 0} \frac{\partial I}{\partial V}\right|_{\Delta T=0} \approx \frac{1}{R}\left\{D_{l}(0) D_{r}(0)+\frac{1}{6}\left(\pi k_{\mathrm{B}} T\right)^{2}\left[D_{l}(0) D_{r}(0)\right]^{\prime \prime}\right\}
\end{aligned}
$$

The thermoelectric power coefficient is then given by

$S=\frac{\pi^{2} k_{\mathrm{B}}^{2} T}{3 e} \frac{\left[D_{l}(0) D_{r}(0)\right]^{\prime}}{D_{l}(0) D_{r}(0)+\frac{1}{6}\left(\pi k_{\mathrm{B}} T\right)^{2}\left[D_{l}(0) D_{r}(0)\right]^{\prime \prime}}$.

Note that, when the second derivative may be ignored, $S$ is proportional to the temperature times the loga-

\#1 As was pointed out by Harrison [9], for an ideal planar junction the tunneling matrix element is proportional to the inverse of the density of states. Here, when we refer to the normalized density of states, we refer to the energy dependent part of the density of states and the tunneling matrix element product. The normalized density of states is then proportional to the square root of the conductivity. 
rithmic derivative of the normalized density of states, which is the known result for bulk metals.

Next, we consider a model junction (motivated by the semiconductor DOS) with electrodes that have the following normalized DOS

$$
\begin{aligned}
D(E) & =D_{\mathrm{b}}, & & E<-\Delta, \\
& =0 & & -\Delta<E<\Delta, \\
& =D_{\mathrm{a}}, & & E>\Delta,
\end{aligned}
$$

where $D_{\mathrm{b}}$ and $D_{\mathrm{a}}$ are the constant normalized density of states below and above the gap of size $2 \Delta$. Since there is a finite gap, the Sommerfeld expansion is not applicable. To proceed, we express the current as

$I=\frac{D_{\mathrm{a}}^{2}}{e R} \int_{e V+\Delta}^{\infty}\left[f^{T}(E-e V)-f^{T+\Delta T}(E)\right] \mathrm{d} E+\frac{D_{\mathrm{b}}^{2}}{e R} \int_{-\infty}^{-\Delta}\left[f^{T}(E-e V)-f^{T+\Delta T}(E)\right] \mathrm{d} E$.

For low temperatures $\left(\Delta \gg k_{\mathrm{B}} T\right)$, eq. (19) is integrated:

$$
\begin{aligned}
I \approx & \frac{D_{\mathrm{a}}^{2}}{e R}\left\{k_{\mathrm{B}} T \exp \left(-\Delta / k_{\mathrm{B}} T\right)-k_{\mathrm{B}}(T+\Delta T) \exp \left[-(e V+\Delta) / k_{\mathrm{B}}(T+\Delta T)\right]\right\} \\
& +\frac{D_{\mathrm{b}}^{2}}{e R}\left\{k_{\mathrm{B}}(T+\Delta T) \exp \left[-\Delta / k_{\mathrm{B}}(T+\Delta T)\right]-k_{\mathrm{B}} T\left[\exp (-e V+\Delta) / k_{\mathrm{B}} T\right]\right\} .
\end{aligned}
$$

The thermoelectric power may now be calculated from eq. (4) to give

$S=-\frac{\left(k_{\mathrm{B}}+\Delta / T\right)\left(D_{\mathrm{b}}^{2}-D_{\mathrm{a}}^{2}\right) \exp \left(-\Delta / k_{\mathrm{B}} T\right)}{e\left(D_{\mathrm{b}}^{2}+D_{\mathrm{a}}^{2}\right) \exp \left(-\Delta / k_{\mathrm{B}} T\right)} \approx \frac{k_{\mathrm{B}}}{e} \frac{D_{\mathrm{a}}^{2}-D_{\mathrm{b}}^{2}}{D_{\mathrm{a}}^{2}+D_{\mathrm{b}}^{2}} \frac{\Delta}{k_{\mathrm{B}} T}$.

In this case, both the electric conductivity $(\partial I / \partial V)$ and the temperature difference contribution to the current $(\partial I / \partial \Delta T)$ are exponentially small with the same exponential dependence $\left(\propto \exp \left(-\Delta / k_{\mathrm{B}} T\right)\right)$. Therefore, $S$ is proportional to the ratio of the prefactors. This result is qualitatively different if there are allowed energy states in the gap region. For example, if there is a small DOS in the gap region, the numerator of eq. (21) will still be exponentially small for low temperatures, but the denominator will contain algebraic terms in $1 / T$. Hence, the exponential terms will no longer cancel and $S$ will exponentially decrease as $T \rightarrow 0$. For a finite DOS in the gap such that

$$
\begin{aligned}
D(E) & =D_{\mathrm{b}}, & & E<-\Delta, \\
& =D_{\mathfrak{g}}, & & -\Delta<E<\Delta, \\
& =D_{\mathrm{a}}, & & E>\Delta,
\end{aligned}
$$

the thermoelectric power coefficient is

$$
S=\frac{\Delta}{e T}\left\{1+\left(k_{\mathrm{B}} T / \Delta\right)\left[\exp \left(\Delta / k_{\mathrm{B}} T\right)+1\right] \ln \left[\exp \left(-\Delta / k_{\mathrm{B}} T\right)+1\right]\right\} \frac{D_{\mathrm{a}}^{2}-D_{\mathrm{b}}^{2}}{D_{\mathrm{a}}^{2}+D_{\mathrm{b}}^{2}+D_{\mathrm{g}}^{2}\left[\exp \left(\Delta / k_{\mathrm{B}} T\right)-1\right]}
$$

In fig. 1 we show the effect of $D_{\mathrm{g}}$ on the temperature dependence of $S$.

For a hypothetical junction made from electrodes with Efros-Shlovsky-like density of states [10] (that is, a parabolic dip near the Fermi level), $S$ decreases exponentially with $T$ for low temperatures. It results from the fact that while $\partial I / \partial \Delta T$ is exponentially small, the conductivity $\partial I / \partial V$ is finite. For an intermediate range 


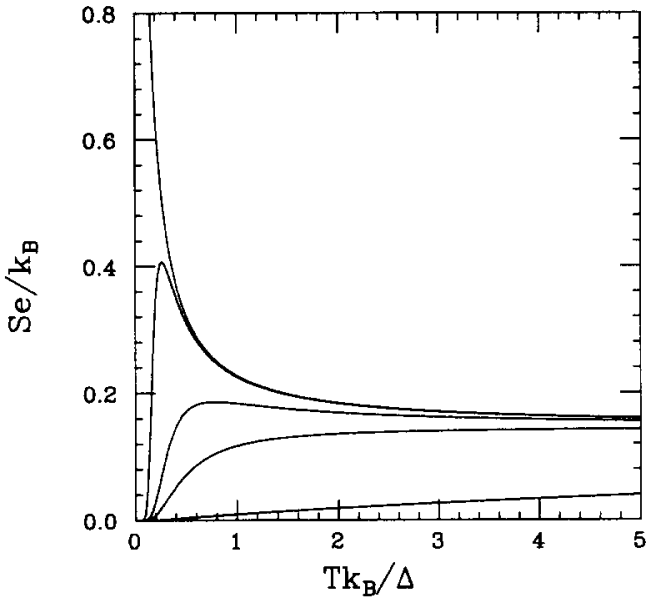

Fig. 1. Calculated thermoelectric power as a function of temperature for a macroscopic junction made from materials with a gaplike structure in the density of states. From the upper curve to the lower curve the values for $D_{\mathrm{g}} / D_{\mathrm{a}}$ in eq. (23) are $0,0.1,0.5,1$ and 5. The value of $D_{\mathrm{b}} / D_{\mathrm{a}}$ is 0.9 for all the curves.

of temperatures, $S$ increases algebraically with decreasing temperature and for high $T, S$ is linear in $T$. (As long as $k_{\mathrm{B}} T$ is much smaller than the electrode work function.)

\section{Thermo-Coulomb effects in mesoscopic normal tunnel junctions}

The response of a mesoscopic normal tunnel junction strongly depends on its coupling to the "external world" (the impedance of the external driving or measuring circuit). For example, the $I-V$ characteristic of the current driven junction is qualitatively different from that of a voltage biased junction [5]. Consequently, the standard Onsager picture

$J_{1}=L_{11} F_{1}+L_{12} F_{2}, \quad J_{2}=L_{21} F_{1}+L_{22} F_{2}, \quad L_{12}=L_{21}$

( $J_{i}$ are the generalized fluxes and $F_{i}$ are the generalized forces) holds only in the limit of "forces control". For the thermoelectric effects we have

$J_{1}=I / e, \quad F_{1}=(1 / T) \Delta \mu=e V / T, \quad J_{2}=I_{\mathrm{Q}}, \quad F_{2}=\Delta(1 / T)$,

where $\mu$ is the electrode chemical potential. These relations hold for voltage and temperature gradient bias. We emphasize that both the thermoelectric power and Peltier coefficients are originally defined for current control. (An open junction may be viewed as current controlled with $I=0$.) For mesoscopic junctions, there is no unique definition for $S$ and $\Pi$, and each possible definition (which yields different temperature dependence of the coefficients) depends on the measurement configuration.

We proceed now to calculate the thermoelectric power coefficient for three limits. First, following the original definition for $S$, we obtain

$S \equiv \frac{\langle V\rangle_{\text {time }}}{\Delta T}$

Here $\langle V\rangle_{\text {time }}$ is the time averaged voltage of an open circuit junction. This case is equivalent to a zero current source, that corresponds to the limit [5]

$\tau_{\mathrm{T}}<\tau_{\mathrm{D}}<\tau_{\mathrm{R}}$,

where $\tau_{\mathrm{T}}$ is the time of the tunneling [11,12], $\tau_{\mathrm{D}}$ is the dwell time (the time between tunneling events), and 
$\tau_{\mathrm{R}}$ is the response time of the external circuit (infinite in this case). The average voltage is given by

$\frac{C\langle V\rangle_{\text {time }}}{e}=\langle n\rangle=\sum_{n=-\infty}^{\infty} n \rho_{\mathrm{eq}}(n)$,

where $\langle n\rangle$ is the average number of charges that have tunneled across the junction (in the direction of the temperature gradient). $\rho_{\mathrm{eq}}(n)$ is the steady state solution of the master equation

$\frac{\partial \rho(n, t)}{\partial t}=r(n+1) \rho(n+1, t)+l(n-1) \rho(n-1, t)-[r(n)+l(n)] \rho(n, t)$.

where the electron tunneling rates are

$r(n)=\frac{1}{e^{2} R} \int_{-\infty}^{\infty} D_{l}\left(E-E_{\mathrm{C}}\right) D_{r}\left(E-2 n E_{\mathrm{C}}\right) f^{T}\left(E-2 n E_{\mathrm{C}}\right)\left[1-f^{T+\Delta T}\left(E-E_{\mathrm{C}}\right)\right] \mathrm{d} E$,
$l(n)=\frac{1}{e^{2} R} \int_{-\infty}^{\infty} D_{l}(E) D_{r}\left(E-2 n E_{\mathrm{C}}-E_{\mathrm{C}}\right) f^{T+\Delta T}(E)\left[1-f^{T}\left(E-2 n E_{\mathrm{C}}-E_{\mathrm{C}}\right)\right] \mathrm{d} E$.

Following Amman et al. [13], $\rho_{\mathrm{eq}}(n)$ is given by

$\rho_{\mathrm{eq}}(n)=\left(\prod_{i=-\infty}^{n-1} l(i)\right)\left(\prod_{i=n+1}^{\infty} r(i)\right)\left[\sum_{i=-\infty}^{\infty}\left(\prod_{i=-\infty}^{j-1} l(i)\right)\left(\prod_{i=j+1}^{\infty} r(i)\right)\right]^{-1}$.

In this limit, the charging energy reduces the tunneling rates, and hence $S$ decreases exponentially at low temperatures, as shown in fig. 2.

The thermoelectric power may alternatively be interpreted as the imposed voltage required for $I=0$ when a temperature difference of $\Delta T$ is applied. When an ideal voltage source is assumed $\left(\tau_{\mathrm{R}}\right.$ is smaller than $\tau_{\mathrm{T}}$ and $\tau_{\mathrm{D}}$, or zero external impedance), the external circuit shorts the capacitance and $S$ is the same as for a macroscopic junction (eq. (17)).

A more interesting case is that of a dwell coupling [5] (intermediate external impedance), that is $\tau_{\mathrm{T}}<\tau_{\mathrm{R}}<\tau_{\mathrm{D}}$

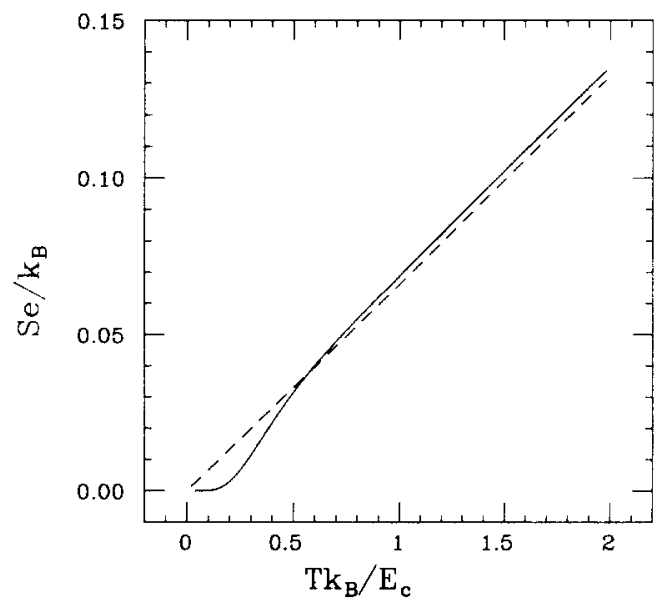

394
Fig. 2. Solid line: calculated thermoelectric power as a function of temperature for a mesoscopic junction in the open junction limit. The electrode normalized density of states is assumed to be linear, $D(E)=D_{1}+D_{2} E / E_{\mathrm{C}}$, with $D_{2} / D_{1}=0.01$. The calculation is made for a fixed ratio $\Delta T / T=0.01$. Dashed line: calculated thermoelectric power for the same system as the solid line except the tunneling rates used in the calculation do not include the charging energy. 
This limit may be either a closed circuit junction with a non-ideal voltage source or an open circuit junction with a non-0ideal voltmeter. To calculate the thermoelectric power coefficient we use the definition of $S$ in eq. (4), $I=e(r-l)$, and the transition rates

$r=\frac{1}{e^{2} R} \int_{-\infty}^{\infty} D_{l}\left(E-E_{\mathrm{C}}\right) D_{r}(E-e V) f^{T}(E-e V)\left[1-f^{T+\Delta T}\left(E-E_{\mathrm{C}}\right)\right] \mathrm{d} E$,

$l=\frac{1}{e^{2} R} \int_{-\infty}^{\infty} D_{l}(E) D_{r}\left(E-e V-E_{\mathrm{C}}\right) f^{T+\Delta T}(E)\left[1-f^{T}\left(E-e V-E_{\mathrm{C}}\right)\right] \mathrm{d} E$.

In the limit $E_{\mathrm{C}} \ll k_{\mathrm{B}} T, S$ is given by eq. (17). In the opposite limit we approximate $f^{T}(E-e V)$ $\times\left[1-\mathrm{f}^{T+\Delta T}\left(\mathrm{E}-\mathrm{E}_{\mathrm{C}}\right)\right]$ in the equation for $r$ as a constant $h$ in the range $e V<E<E_{\mathrm{C}}$,

$h \approx \exp \left(-\frac{E-e V}{k_{\mathrm{B}} T}\right) \exp \left(\frac{E-E_{\mathrm{C}}}{k_{\mathrm{B}}(T+\Delta T)}\right)$.

Using this approximation and a similar one for $l$ we get

$S \approx \frac{k_{\mathrm{B}}}{e} H\left(E_{\mathrm{C}}\right) \frac{E_{\mathrm{C}}}{k_{\mathrm{B}} T}$,

where

$$
\begin{aligned}
& H\left(E_{\mathrm{C}}\right)=\int_{0}^{E_{\mathrm{C}}}\left[D_{l}\left(E-E_{\mathrm{C}}\right) D_{r}(E)\left(E / E_{\mathrm{C}}-1\right)+D_{l}(E) D_{r}\left(E-E_{\mathrm{C}}\right) E / E_{\mathrm{C}}\right] \mathrm{d} E \\
& \quad \times\left(\int_{0}^{E_{\mathrm{C}}}\left[D_{l}\left(E-E_{\mathrm{C}}\right) D_{r}(E)+D_{l}(E) D_{r}\left(E-E_{\mathrm{C}}\right)\right] \mathrm{d} E\right)^{-1} .
\end{aligned}
$$

Therefore, in this limit, the thermoelectric power at low temperatures is gap-like with the gap replaced by the charging energy.

\section{A comment about the Peltier effect}

For macroscopic junctions the Peltier coefficient $\Pi$ is directly obtained from $S$, using eq. (8). We emphasize again that $\Pi$ is proportional to the average energy carried by the tunneling charges. Hence, the heat current $I_{\mathrm{Q}}$ differs from the Joule heat which is a measure of the heat production rate (due to the current $I$ ).

For mesoscopic junctions eq. ( 8 ) does not hold, and $\Pi$ has to be derived separately. The original definition of the Peltier effect implicitly assumes the current source limit. In this limit, the entropy production due to the charge tunneling (assuming the electron tunnels from the Fermi energy level in the direction of the voltage drop) is

$\Delta S=\frac{e V_{\mathbf{T}}-e^{2} / 2 C}{T}$,

where $V_{\mathrm{T}}$ is the voltage before the tunneling event. Hence, $\langle\Delta S\rangle=e\langle V\rangle / T$ and the Joule heating is $I\langle V\rangle$ (as expected).

In a mesoscopic junction, when an electron tunnels, the electric field of the junction acts on the electron, but this field changes as the electron tunnels so that $\left\langle I_{\mathrm{Q}}\right\rangle$ and $\langle I\rangle$ are no longer simply related. A more detailed discussion of this point and the time dependence of $\left\langle I_{Q}\right\rangle$ will be presented in a forthcoming publication [14]. 


\section{Conclusions}

We have demonstrated that the thermoelectric effects provide a very sensitive method of studying the normalized DOS structure and the interaction of the mesoscopic junction with the "external field". In recent years the scanning tunneling microscope (STM), and especially the CSTM (cryogenic STM), have been used for spectroscopic measurements (measurements of the local DOS) in addition to topographic measurements. Wilkins et al. [15] have shown that charging effects (Coulomb blockade) due to single charge transfer via oxide impurities may obscure the measurement of the DOS and lead to a wrong interpretation (e.g. they can lead to a gap-like structure in the DOS). The results presented here suggest a method to overcome these difficulties by the complementary measurement (to the $I-V$ characteristic) of the thermoelectric power coefficient. Recently Williams and Wickramasinghe [16] have performed thermoelectric power measurements at relatively large temperatures, followed by the theoretical study of Stovneng and Lipavsky [17]. Leavens and Aers [18] presented a pioneering study of "vacuum tunneling thermopower" with focus on the effect of the image potential.

In a forthcoming publication we show that the sensitivity can be further increased by using two serially coupled junctions [14]. It is also shown that the thermoelectric effects provide an excellent method to study resonant tunneling versus consecutive tunneling in serially connected tunnel barriers, as well as dephasing between tunneling events.

\section{Acknowledgement}

We have benefited from enlightening discussions with L.C. Davis on the theory of tunneling and thermoelectric power. We would also like to thank Y. Gefen, R.C. Jaklevic, A. Stern and R. Wilkins for useful discussions. This research was partially supported by the U.S. Army Research Office under the University Research Initiative program, Contract No. DAAL03-87-K-007, and the Wolfson Foundation through the Israel Academy of Science.

\section{References}

[1] D.V. Averin and K.K. Likharev, in: Mesoscopic phenomena in solids, eds. B.L. Altshuler, P.A. Lee and R.A. Webb (Elsevier, Amsterdam, 1991).

[2] P. Delsing, D.B. Haviland, T. Claeson, A.N. Korotov and K.K. Liharev, in: SQUID 91, eds. H. Koch and H. Lubbig (Springer, Berlin, 1992), and references therein;

M. Amman, E. Ben-Jacob and Z. Hermon, in: SQUID 91, eds. H. Koch and H. Lubbig (Springer, Berlin, 1992), and references therein.

[3] J.E. Mooij, B.J. van Wees, L.J. Geerligs, M. Peters, R. Fazio and G. Schon, Phys. Rev. Lett. 65 (1990) 645.

[4] M.H. Devoret, D. Esteve, H. Grabert, G.-L. Ingold, H. Pothier and C. Urbina, Phys. Rev. Lett. 64 (1990) 1824.

[5] M. Amman, R. Wilkins, E. Ben-Jacob and R.C. Jaklevic, to be published.

[6] M. Amman, E. Ben-Jacob and J.L. Cohn, Z. Phys. B 85 (1991) 405.

[7] H.B. Callen, Phys. Rev. 73 (1948) 1349.

[8] E.L. Wolf, Principles of electron tunneling spectroscopy (Oxford Univ. Press, Oxford, 1985).

[9] W.A. Harrison, Phys. Rev. 123 (1961) 85.

[10] A.L. Efros and B.I. Shklovskii, J. Phys. C 8 (1975) L49.

[11] M. Buttiker and R. Landauer, Phys. Rev. Lett. 49 (1982) 1739.

[12] E.H. Hauge and J.A. Stovneng, Rev. Mod. Phys. 61 (1989) 917.

[13] M. Amman, R. Wilkins, E. Ben-Jacob, P.D. Maker and R.C Jaklevic, Phys. Rev. B 43 (1991) 1146.

[14] E. Ben-Jacob and M. Frank, to be published.

[15] R. Wilkins, M. Amman, E. Ben-Jacob and R.C. Jaklevic, Phys. Rev. B 42 (1990) 8698.

[16] C.C. Williams and H.K. Wickramasinghe, Nature 344 (1990) 317.

[17] J.A. Stovneng and P. Lipavsky, Phys. Rev. B 42 (1990) 9214.

[18] C.R. Leavens and G.C. Aers, Solid State Commun. 61 (1987) 289. 